"This document is the Accepted Manuscript version of a Published Work that appeared in final form in Journal of the American Chemical Society, copyright (c) American Chemical Society after peer review and technical editing by the publisher. To access the final edited and published work see http://pubs.acs.org/doi/pdf/10.1021/ja801968f 


\title{
Photo-Switched DNA-Binding of a Photochromic Spiropyran
}

\author{
Johanna Andersson, Shiming Li, Per Lincoln, and Joakim Andréasson*
}

Department of Chemical and Biological Engineering, Physical Chemistry,

Chalmers University of Technology, SE-41296 Göteborg, Sweden

RECEIVED DATE (automatically inserted by publisher); E-mail: a-son@chalmers.se

One of the many challenges of chemical biology is the design and the understanding of the mechanisms of small DNA-binding molecules. ${ }^{1,2}$ Since most of the DNA-associated processes occurring in the cell could be inhibited by a molecule with a high DNA-binding affinity, controlling the action using external stimuli would be of tremendous utility for e.g. chemotherapeutics. ${ }^{3,4}$ Ultimately, a molecule for this purpose should show no or very small affinity for the DNA double helix in the "passive" form. Upon activation the molecule should interconvert to a form that binds strongly to DNA to inhibit the targeted process. Here, we report a photochromic molecule from the $\operatorname{spiropyran}^{5}$ (SP) family that can be activated using UV light from a form that shows no signs of interaction with DNA to a form that binds to DNA by intercalation. The corresponding deactivation can be achieved by visible light, i.e., the DNAbinding process is fully reversible using light as the only stimulus. The idea of switching the state of photochromes in order to control various DNA-associated processes has been demonstrated before. $^{6-13}$ However, in the vast majority of these pioneering studies, the approach has been to covalently link photochromic units to short single-stranded oligonucleotides ${ }^{6-12}$, while other authors have been targeting mismatch-containing $11-$ mers $^{13}$. Our approach surmounts the need of covalently modifying the targeted DNA molecules and the principle is applicable on naturally occurring DNA, making it much more suitable for any practical biological application.

Chart 1 shows the structures and the isomerization scheme of photochromic spiropyran $\mathbf{1}$ used in this work. The thermally stable spiro form 1c is isomerized to the ring-opened merocyanine form 10 by exposure to UV light. Shown in Chart 1 is the transtrans-cis form of 10, which has been suggested to be the thermodynamically stable 1o-isomer. ${ }^{14-16}$ The photo-stationary distribution after exposure to $254 \mathrm{~nm}$ light is ca. $54 / 46 \mathbf{1 c} / \mathbf{1 o}$ as judged by NMR measurements whereas visible light $(600>\lambda>475$ $\mathrm{nm}, \sim 10 \mathrm{~mW} / \mathrm{cm}^{2}$ ) converts the sample to $100 \% \mathbf{1 c}$ with a time constant of $\sim 1 \mathrm{~min}$. By contrast, the time constant for establishment of thermal equilibrium at $23{ }^{\circ} \mathrm{C}$ is $124 \mathrm{~min}$. The equilibrium composition is ca. $67 / 33 \mathrm{1c} / 1 \mathrm{o}$.

Chart 1. Structures of photochromic spiropyran 1

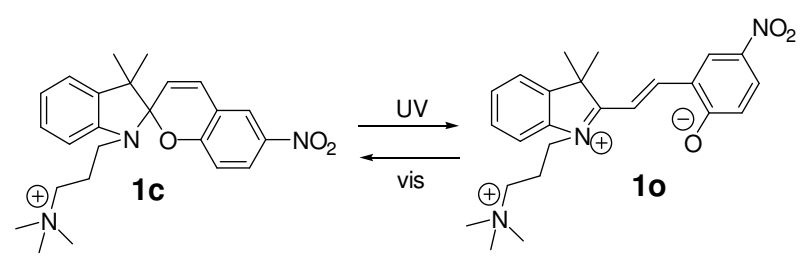

As shown in Figure 1, 1c does not absorb light significantly in the visible region; its longest-wavelength absorption band is at $348 \mathrm{~nm}$. 10 displays absorption in the visible region with the strongest absorption band centered at $512 \mathrm{~nm}$.

The absorption spectra of $\mathbf{1 c}$ with and without addition of calfthymus (CT) DNA are virtually superimposable as illustrated in Figure 1 (top panel, 1c), indicating no or very weak interactions between 1c and DNA. The corresponding spectra of 10 are shown in the lower panel. Here, the spectral changes upon addition of
DNA are dramatic as seen by comparing the spectra of 10 without DNA (blue line) with that in the presence of DNA (red line). In fact, the spectral perturbation is even stronger since some unbound 10 remains in solution. A spectrum representing 100\% DNA-bound 10 (green line) shows that the longest-wavelength band is shifted from $512 \mathrm{~nm}$ to $420 \mathrm{~nm}$, i.e., there is a $92 \mathrm{~nm}$ shift toward shorter wavelengths upon DNA addition. Furthermore, the band has experienced a ca. $45 \%$ hypochromic shift, i.e., a decrease in the absorbance (see Supporting Information for details on the spectral deconvolutions). These observations clearly show that 10 interacts strongly with DNA.

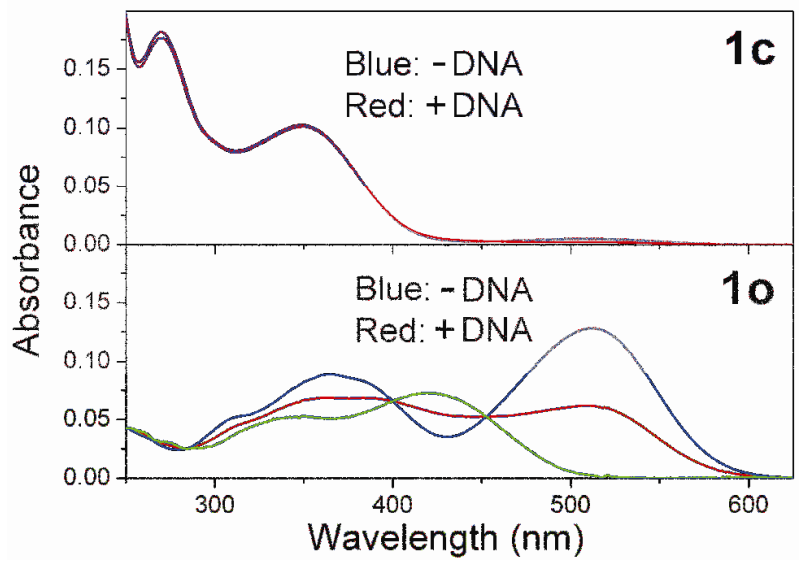

Figure 1. Absorption spectra of 1c (top panel) and $\mathbf{1 o}$ (lower panel) in the absence (blue lines) and presence (red lines) of calf-thymus DNA. The contribution from DNA to the overall absorption has been subtracted for ease of comparison. Likewise, the contribution from 1c has been subtracted from the spectra of $\mathbf{1 0}$ shown in the lower panel. The green line corresponds to a sample of $100 \% 10$ bound to DNA as the contribution from unbound 10 has been corrected for (see Supporting Information for details). The total concentration of $\mathbf{1}$ was approximately $1.5 \times 10^{-5} \mathrm{M}$. The concentration of DNA was $11.6 \times 10^{-5} \mathrm{M}$, and the $\mathrm{NaCl}$-concentration of the solution was $8.6 \times 10^{-3} \mathrm{M}$.

Figure 2 shows the results from flow-oriented linear dichroism (LD) measurements ${ }^{17}$ where 1 was exposed to alternating UV and visible light irradiation in presence of calf-thymus DNA. The first spectrum was recorded on a sample containing 1c and DNA and shows a weak negative LD signal in the visible, originating from a small fraction of thermally formed 1o. UV irradiation of the sample for 5 minutes converted a substantial fraction to the open form 1o, and the isomerization process is paralleled by an amplification of the negative LD signal by more than a factor 3 . Subsequent exposure to visible light for 5 minutes decreased the LD response to almost the initial value, signaling that the DNAbinding process can be light-switched both ways. Indeed, upon exposure to a second 5 minutes portion of UV light the previous high-amplitude value of the LD signal was nearly recovered, proving that the observed changes in the LD signal are due to a fully reversible, light-controlled DNA-binding process rather than photo-decomposition. As seen in the inset, the bands of the LD spectra virtually coincide with the absorption bands of DNAbound 10. 


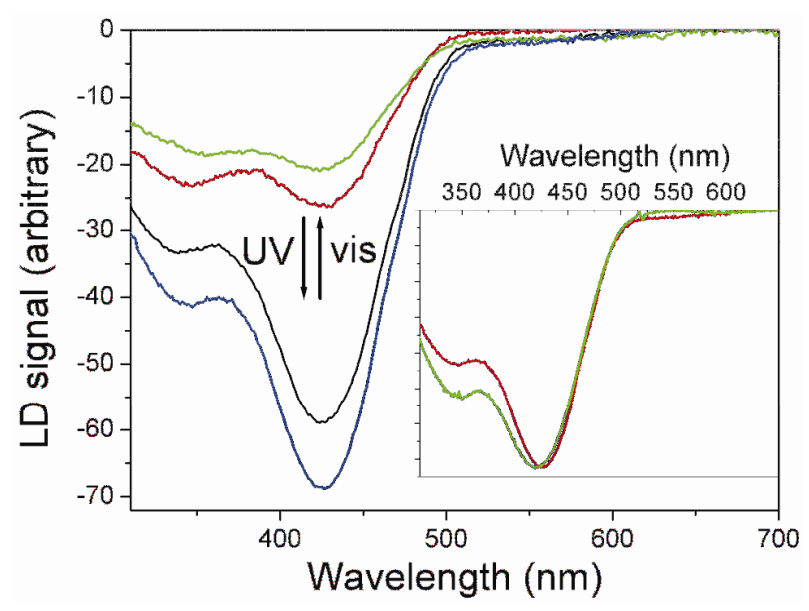

Figure 2. Flow-oriented LD spectra on a sample of $\mathbf{1}$ and calf-thymus DNA after the following irradiation sequence: no light exposure (green line), 5 minutes UV irradiation (blue line), 5 minutes visible light irradiation (red line), and 5 more minutes UV irradiation (black line). The inset shows the LD signal observed for 1o + DNA (red line) and the absorption spectrum of DNA-bound 10 (green line). The negative of the absorption spectrum is shown for ease of comparison. The total concentration of 1 was approximately $9 \times 10^{-5} \mathrm{M}$. The concentration of DNA was $13.3 \times 10^{-5} \mathrm{M}$, and the $\mathrm{NaCl}$-concentration of the solution was $8.6 \times 10^{-3} \mathrm{M}$.

The reduced LD (LD/Abs) may be analyzed in terms of the angle between the transition moment of the DNA-binding molecule and the DNA helix axis to provide information about binding geometries. ${ }^{18}$ Here it was found that the angle between the transition of 10 centered at $420 \mathrm{~nm}$ and the helix axis is $80 \pm 5$ degrees, which is a strong indication for intercalation (see Supporting information for details).

Upon intercalation of a reagent between the DNA-bases, the absorption of the reagent typically experiences a hypochromic shift. In Figure 1 it is seen that the absorption of $\mathbf{1 0}$ is indeed decreased by $45 \%$ upon binding to DNA. Furthermore, the spectrum is shifted $92 \mathrm{~nm}$ toward shorter wavelengths. This observation could be explained by inspecting the two different resonance structures of 10. The zwitterionic structure shown in Chart 1 is accompanied by a resonance structure where the nitrogen in the heterocycle and the phenolic oxygen both are formally charge neutral. This form should be favored in a hydrophobic environment, e.g., between the DNA-bases, whereas the zwitterionic form should be more likely to find in a highly polar medium such as water. This notion is supported by the fact that in chloroform, where the formally charge neutral structure is more likely, the longest-wavelength absorption band of $\mathbf{1 0}$ is centered at $438 \mathrm{~nm}$ (not shown). The corresponding value in water is $512 \mathrm{~nm}$, i.e., there is a $74 \mathrm{~nm}$ shift toward shorter wavelengths in going from a highly polar water environment to less polar chloroform. ${ }^{19}$ The pronounced solvatochromism for 10 makes it an excellent candidate for a nucleic acid probe, as the color changes drastically upon changes in the polarity of the environment.
The absorption changes observed upon addition of DNA shown in Figure 1 can also be used to roughly estimate the binding constant for 10 (see Supporting Information). The resulting number of ca. $2 \times 10^{4} \mathrm{M}^{-1}$ is not very high, but would be more than sufficient for applications where the difference in binding affinity between $1 \mathrm{c}$ and $1 \mathrm{o}$ is the key parameter. A comparison between the absorption spectra of 10 recorded in the presence of AT-DNA $\left([\operatorname{poly}(\mathrm{dA}-\mathrm{dT})]_{2}\right)$ and GC-DNA ([poly $\left.\left.(\mathrm{dG}-\mathrm{dC})\right]_{2}\right)$ shows that the binding affinity of 10 increases in the following order: GCDNA $<$ CT-DNA<AT-DNA (see Supporting Information for details).

In conclusion, we have demonstrated the dramatically different DNA-binding properties of the two isomeric forms of a photochromic spiropyran. The closed, non-binding form is activated by UV light to the open form that intercalates the DNAbases. The open form in turn can be deactivated and dissociated by visible light, making the DNA-binding equilibrium completely tunable by light.

Acknowledgement. This work was supported by the Swedish Research Council (VR).

Supporting Information Available: Details on the synthesis, the experimental procedures, the deconvolution of the absorption spectra, and the DNA-binding studies. This material is available free of charge via the Internet at http://pubs.acs.org.

\section{References}

(1) Dervan, P. B. Bioorg. Med. Chem. 2001, 9, 2215-2235

(2) Nelson, S. M.; Ferguson, L. R.; Denny, W. A. Mutat. Res. 2007, 623, $24-$ 40.

(3) Willner, I. Acc. Chem. Res. 1997, 30, 347-356.

(4) Mayer, G.; Heckel, A. Angew. Chem. Int. Ed. 2006, 45, 4900-4921.

(5) Bertelson, R. C. In Organic Photochromic and Thermochromic Compounds; Crano, J. C., Guglielmetti, R. J., Eds.; Plenum Press: New Compounds; Crano, J. C., Guglic
York, 1998; Vol. 1, pp 11-83.

(6) Asanuma, H.; Ito, T.; Yoshida, T.; Liang, X.; Komiyama, M. Angew. Chem. Int. Ed. 1999, 38, 2393-2395.

(7) Nishioka, H.; Liang, X.; Kashida, H.; Asanuma, H. Chem. Commun. 2007, 4354-4356.

(8) Asanuma, H.; Takarada, T.; Yoshida, T.; Tamaru, D.; Liang, X.; Komiyama, M. Angew. Chem. Int. Ed. 2001, 40, 2671-2673.

(9) Liu, M.; Asanuma, H.; Komiyama, M. J. Am. Chem. Soc. 2006, 128, 1009-1015.

(10) Yamazawa, A.; Liang, X.; Asanuma, H.; Komiyama, M. Angew. Chem. Int. Ed. 2000, 39, 2356-2357.

(11) Liang, X.; Asanuma, H.; Komiyama, M. J. Am. Chem. Soc. 2002, 124, 1877-1883.

(12) Matsunaga, D.; Asanuma, H.; Komiyama, M. J. Am. Chem. Soc. 2004, $126,11452-11453$

(13) Dohno, C.; Uno, S.; Nakatani, K. J. Am. Chem. Soc. 2007, 129, 1189811899.

(14) Aubard, J. In Organic Photochromic and Thermochromic Compounds; Crano, J. C., Guglielmetti, R. J., Eds.; Kluwer Academic Publishers: Hingham, MA, 1999; Vol. 2, pp 369-370.

(15) Sheng, Y.; Leszcynski, J.; Garcia, A. G.; Rosario, R.; Gust, D.; Springer, J. J. Phys. Chem. B 2004, 108, 16233-16243.

(16) Wohl, C. J.; Kuciauskas, D. J. Phys. Chem. B 2005, 109, 22186-22191.

(17) Nordén, B.; Tjerneld, F. Biophys. Chem. 1976, 4, 191-198.

(18) Nordén, B.; Kubista, M.; Kurucsev, T. Quart. Rev. Biophys. 1992, 25, 51170.

(19) As the wavelength shift upon intercalation is larger than the corresponding shift in going from water to chloroform, it is likely that the intercalation process induces a conformational change to a more nonplanar structure of 10 compared to solution. 


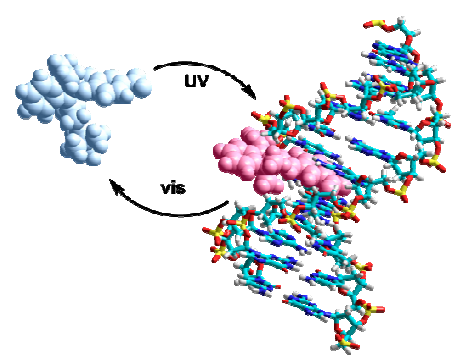

The dramatically different DNA-binding properties of the two isomeric forms of a photochromic spiropyran have been demonstrated, enabling photo-switched DNA-binding. The closed, UV-absorbing form shows no signs of interaction with DNA. Upon UV exposure the spiropyran is isomerized to the open form that binds to DNA by intercalation. The process is fully reversible as the corresponding dissociation process is induced by visible light. 